\title{
Compression sleeves design based on Laplace laws
}

\begin{abstract}
Compression sleeves are very important to the prevention and control of human limb hyperplasia scaring. The garment pressure is the key determinant of the curative effect, and one of the key factors which influence this value is the precision of Compression sleeves design. In order to study the design and performance of Compression sleeves via relatively simple but accurate methodologies, different deformation formulas of Laplace law were summarized and analyzed. Firstly, it was found that both of the deformation formulas of Laplace law proposed by Maklewska and Leung respectively were based on the principle that the arm radius was calculated by the arm circumference, furthermore, the arm radius, fabric tensile index and the preset clothing pressure were substituted into the Laplace law formula, and the sizes of the Compression sleeves could be calculated. Since the design methods proposed above were easier to implement in practical production, Compression sleeves were designed and manufactured based on the deformation formulas of Laplace law, and garment pressures were tested and compared with the preset pressures. Experimental results showed that garment pressures of the Compression sleeves which were designed based on the Maklewska Laplace law within the scope of the required pressure value, so this deformation could be used for designing the Compression sleeves accurately. However, the sizes of the Compression sleeves which were calculated based on the Leung Laplace law were too small to put on, so the formula of Leung Laplace law was not suitable for designing the Compression sleeves.
\end{abstract}

Keywords: laplace law, compression sleeves, reduction factor, garment pressure
Volume 2 Issue 2 - 2017

\author{
Lihuan Zhao, Xiaohuan Li, Jie Yu, Cuiyu Li, \\ Guangxu Li \\ Tianjin Polytechnic University, China
}

Correspondence: Lihuan Zhao, School of Textiles, Tianjin Polytechnic University, West Binshui Road 399, Xiqing District, Tianjin, 300387, China, Tel 02283955430 .

Email zhaolihuan@tjpu.edu.cn

Received: April 22, 2017| Published: June 20, 2017

\section{Introduction}

Compression garments are often used for preventing and controlling the excessive proliferation of scar tissue, which usually occurs after burn, surgical trauma. ${ }^{1}$ Hyperplasia scar appears in the early healing, especially during the breeding granulation periods. At present, more consistent view about the Hyperplasia scar is that the Hyperplasia scar is caused by excessive fibroblast proliferation and the imbalance of the extracellular matrix's synthesis and degradation. ${ }^{2}$ There are many methods for the treatment of the Hyperplasia scar, such as pressure therapy, intralesional corticosteroids, radiation therapy and topical medications. ${ }^{3}$ Pressure therapy is one of the most effective methods to prevention and treatment of Hyperplasia scar and this method is easy to operate, having an exact curative effect, low price, and is noninvasive treatment. ${ }^{4,5}$

The main principle of the pressure therapy is that the special pressure (i.e., the garment pressure) produced by the compression garment can restrict blood flow to scar area and prevent the growth of the Hyperplasia scar tissue, thereby limiting the formation of Hyperplasia scar. ${ }^{6}$ Therefore the value of the pressure delivered by the compression garment on the Hyperplasia scar part is the key determinant of the curative effect. Garment pressure refers to the unit pressure exerted by the elastic garment (such as compression garment, pantyhose, etc.) on the human skin surface and the unit of the pressure is $\mathrm{mmHg}$ or $\mathrm{Pa}^{7}$ Generally, the pressure exerted by the compression garment on the Hyperplasia part should not be too small or too large. If too small, it will not have curative effect, while if too large, it may occur some pathological response such as blood circulation obstacle, accelerated breathing, indigestion and internal organs deformation includes the gastroptosis. ${ }^{8}$ For the children, the large pressure will be more serious which might affect the development of their local bone and even cause deformity. ${ }^{9}$ Therefore, for the application of compression garments, the key factor is how to design the compression garment's sizes to achieve the required pressure exerted on the body part.

The level of garment pressure depends on the physical and mechanical properties of the clothing, the shape and structure characteristics of the human body exerted pressure and the accuracy of the sizes of compression garment. So, the only key factor affects the garment pressure is the accuracy of the sizes of compression garment, in the case of the first two factors are certain. Many researchers have done a lot of work on designing of the compression garments. At present, the main methods for designing the compression garments include the reduction factor, the Laplace law and the numerical simulation methods. The reduction factor method refers to select a standard reduction factor (generally $10 \%, 15 \%$ or $20 \%$ ) firstly, and then calculate the sizes of compression garment based on the sizes of the wound part of human body. The compression garment will impose pressure on the body part since the sizes of garment are smaller than those of the body's. However, the reduction factor used in this method is relatively in an arbitrary manner since it will not change with the particular body part or different properties of fabrics. Therefore, compression garments designed by the reduction factor method could not exert required pressure on the particular human body. Numerical simulation method combines the digital simulation technology of garment pressure of compression garment with objective tests, so the pressure magnitudes and distribution can be obtained by calculating before the real garment is manufactured. The precision of the sizes of compression garment calculated by this method mainly depend on the accuracy of the models for compression garment and human body and the numerical simulation. Moreover, this method is difficult to implement since its cost is relatively higher. The design method 
for compression garment based on Laplace law considers the fabric properties as well as the body sizes, the accuracy of the sizes of compression garment made by this method may not as precise as numerical simulation method, but its accuracy is much higher than that of reduction factor method's; what is more, Laplace law method is easy to implement, having low cost and high popularization value.

The aim of the study is to obtain relatively simple (body sizes obtained by the circumstances of the corresponding parts) and accurate methods to design the compression sleeves (human arm is one of the relatively concentrated wound areas) by analyzing the existing researches of designing compression garments or pressure bandages based on Laplace law. Therefore, various methods for designing of compression garments or pressure bandages based on the Laplace law or its deformation formulas were analyzed to find out the relatively simple and accuracy formulas firstly; and then, compression sleeves were made based on the formulas and the garment pressures were tested to verify the accuracy and applicability of the formulas. This study can provide solutions for precisely designing compression sleeves and forecast research results will also be useful for solving the common problems of accurately designing elastic garments used for other fields as well (shaping underwear, bras, varicose veins tights, knee and arm guards, etc.).

\section{The methods for designing compression gar- ments or compression bandages based on Laplace law}

\section{The theory of the laplace law}

The Laplace law was originally developed by Thomas Young and Pierre Simon de Laplace in 1806 to explain the surface tension phenomenon in liquids. The equation could be written as formula (1).

$$
P_{\alpha}-P_{\beta}=\frac{2 T}{R}
$$

Where, $\mathrm{P}_{\alpha}$ and $\mathrm{P}_{\beta}$ were the internal and external pressure of the liquid respectively $(\mathrm{Pa})$, T was the tension of the liquid surface $(\mathrm{N} / \mathrm{m})$, $\mathrm{R}$ was the radius of curvature $(\mathrm{m})$. This equation suggested that the internal pressure was often bigger than the external pressure, however, when the R was infinitely great, that was to say when the spherical surface tended to plane, the differences between internal and external pressure tended to be zero.

When calculated cylindrical model surface pressure, the above equation was modified as follows:

$$
P=\frac{T}{R}
$$

Where, $\mathrm{P}$ was cylindrical surface pressure $(\mathrm{Pa}), \mathrm{T}$ was cylindrical surface tension (for fabric, the tension referred to per meter's fabric tension) $(\mathrm{N} / \mathrm{m})$, R was the radius of curvature of the cylindrical surface $(\mathrm{m})$.

Cheng et al. ${ }^{10}$ studied the relationship between the garment pressure and the body sizes, and found that with the increase of human body sizes, the pressure value measured decreased; at the same time, with the decrease of human body sizes, the pressure value measured increased, and they thought that this phenomenon might be explained by Laplace law.

\section{Deformation formulas of laplace law}

Laplace law assumed that the human body was a cylinder; however, the body contour surface was not regular. In order to better simulate the pressure exerted by the compression garment, the relationship between body contour surface and cylinder surface needed be determined given the same circumference. Therefore, the compression factor $\mathrm{CF}$ was introduced combined with the Laplace law, meanwhile, the fabric elastic modulus and the relationship between the reduction factor and the elongation were considered, $\mathrm{Ng}$ et al. ${ }^{11}$ concluded the following formula:

$$
R_{e}=\frac{1}{1+\frac{2 \pi E I(C F)}{C_{\text {human }} P}}
$$

Where, Re was reduction factor, EI was elastic module, CF was compression factor, $\mathrm{C}_{\text {human }}$ was human body circumference $(\mathrm{cm}), \mathrm{P}$ was garment pressure $\left(\mathrm{gf} / \mathrm{cm}^{2}\right)$.

Calculated the reduction factor according to the formula (3), and calculated the sizes of compression garment based on the relationship between the sizes of body and garment with the calculated reduction factor, and then compression garment was made. The test results proved that the overall variance $\sigma^{2}$ between the tested value and the required pressure was approximately 2.0 , which agreed that the two values were very closely. But the value of CF was influenced by the deformation degree of knitted fabric and the differences among body parts, so this model had some limitations.

Thomas ${ }^{12}$ considered that the pressure exerted by the bandage was influenced by the width and the layer number of the bandage. Therefore the formula (2) was rewritten as follows:

$$
P=\frac{T n}{R W}
$$

Where, $\mathrm{P}$ was pressure $(\mathrm{Pa}), \mathrm{n}$ was the layer of bandage, $\mathrm{W}$ was the width of bandage $(\mathrm{m})$, $\mathrm{T}$ was the fabric tension $(\mathrm{N} / \mathrm{M}), \mathrm{R}$ was the radius of curvature of the limbs (m).

Formula (4) could be further modified to predict the pressure in $\mathrm{mmHg}$, which is the common unit of pressure regarding to the compression garment. When patients are measured for making compression garments in hospital, it is the circumference of the limb or body part that is measured (not the radius). Therefore, the formula (4) could be rewritten as follows:

$$
P=\frac{T \times n \times 4620}{C W}
$$

Where, $\mathrm{P}$ was the bandage pressure $(\mathrm{mmHg}), \mathrm{T}$ was the tension of bandage $(\mathrm{Kgf}), \mathrm{n}$ was the layer of bandage, $\mathrm{C}$ was the circumference of the limb $(\mathrm{cm})$, W was the width of bandage $(\mathrm{cm})$.

Formula (5) improved the precision of the pressure of multilayer bandage calculated by Laplace law. However, this formula just added the pressures of each layer bandages together to represent the pressure of multiple integral bandage, without considering the friction between the layers of bandages and the impact of the arrangement way of the layers on the overall pressure, which resulting certain deviation between the calculation results and the actual pressure. 
Macintyre et al. ${ }^{13}$ also amended the Laplace law, the formula was expressed as follows:

$$
P=\frac{T}{\frac{C}{2 \pi}}=\frac{6.283 T}{C}
$$

Where, $\mathrm{P}$ was the pressure $(\mathrm{Pa}), \mathrm{T}$ was the fabric tension $(\mathrm{N} / \mathrm{m}), \mathrm{R}$ was the radius of curvature of the limbs (m).

If the pressure was represented with the $\mathrm{mmHg}$, the formula could be rewritten as follows:

$$
P=\frac{2 \pi T}{133.322 \mathrm{C}}=\frac{0.047 T}{C}
$$

Where, $\mathrm{P}$ was the pressure $(\mathrm{mmHg})$, $\mathrm{T}$ was the fabric tension $(\mathrm{N} / \mathrm{m}), \mathrm{C}$ was the circumference of the limbs $(\mathrm{m})$.

Macintyre et al made the compression garments for the forearm and the thigh using the most popular fabrics which were often used in hospitals, and the test results showed that the calculated value of Laplace law was $10.9 \mathrm{mmHg}$ higher than the measured value at the forearm. However, the pressure was closely to the calculated value of Laplace law when the garment pressure measured at the thigh. This showed that Laplace law was suitable for predicting pressure on large circumference, and in some cases, Laplace formula might not predict the pressure on small circumference accurately.

Maklewska et al. ${ }^{14}$ deduced the garment pressure based on Laplace law with considering the fabric properties. The formula was represented as follows:

$$
P=\frac{2 \pi F}{G_{1} W}
$$

Where, $\mathrm{F}$ was fabric tension $(\mathrm{cN})$ with the fabric width was $\mathrm{W}$ $(\mathrm{cm}), \mathrm{G}_{1}$ was the circumference of the limbs $(\mathrm{cm}), \mathrm{W}$ was the width of the fabric, $\mathrm{P}$ was pressure $(\mathrm{hPa})$.

Compared with the compression garment designed based on reduction factor method, the pressure of the compression garment designed based on formula (8) was close to the required value, however, the pressure of the compression garment designed based on the reduction factor method fell continuously, which had a large difference from the required value.

Leung et al. ${ }^{15}$ not only considered the type of the elastic fabric and the level of the reduction factor, but also considered the elastic modulus and the anisotropic behavior of fabric. The improved formula had been rewritten as follows:

$$
P=\frac{2 \pi E A_{0} \varepsilon}{133.32 C^{2}}
$$

Where, $\mathrm{P}$ was the pressure $(\mathrm{mmHg}), \mathrm{E}$ was the elastic modulus $(\mathrm{Pa}), \mathrm{A}_{0}$ was the cross-sectional area of fabric $\left(\mathrm{m}^{2}\right), \mathrm{C}$ was the circumference of body part (m).

The modulus might be changed once the bias angles between two layers fabric were changed, so this formula provided lager design space for designing compression garments. However, sometimes each of the two layers might produce bigger or smaller pressure, which resulting the pressure exerted by the two layers would be bigger or less. How to choose just right bias angles for different fabrics was the key to produce the required pressure in this case.

\section{Compression sleeves design based on laplace law}

From the analysis of the basic Laplace law and its deformation formulas, it is found that there are many deformations of Laplace law for improving the accuracy of the garment pressure of compression garment or pressure bandage. However, researches about the applicability of these deformation formulas are less. This study aims to discuss the applicability of the deformation formulas by studying of the design and manufacture methods for compression sleeves based on Laplace law, and the results will also provide the reference for the design and manufacture of other tubular compression garments.

Among the deformation formulas of Laplace law, because of the compression factor and the cylindrical model used in the experiment, formula (3) has poor repeatability; formula (4) and formula (5) take more focus on the influence of pressure bandage layers on the final pressure value. Formula (8) (researched by Maklewska, shorted for FOM) and formula (9) (researched by Leung, shorted for FOL) consider the elastic modulus and tensile properties of the fabrics, and the parameters of FOM or FOL can be obtained through the experiments, and the reproducibility of the results of the two formulas are easy to get. Therefore, this paper aimed at study the applicability of the formula (8) and formula (9) by designing the compression sleeves.

\section{Materials}

At present, the fabrics for making compression garments are provided by the companies, and there are only several mainly kinds of fabrics on the market. In this study, five kinds of samples (named as A, B, C, D and E, respectively) were collected, which were common used by the major hospitals for medical use. The parameters of the five fabrics (samples) were shown in Table 1, and the materials of the samples in Table 1 are Spandex and Nylon.

\section{Tensile test}

Instrument: Instron tensile strength machine 3369.

Experiment parameters: Per-tension was $0.1 \mathrm{~N}$, tensile rate was $100 \mathrm{~mm} / \mathrm{min}$ and gauge length was $100 \mathrm{~mm}$.

Specimen size (lengthways direction): $(200 \times 50) \mathrm{mm}$.

Experiment index: each specimen was tested at a certain elongation, and the elongations were given as follows: $10 \%, 20 \%, 30 \%, 40 \%, 50 \%$ and $60 \%$, and then recorded the tensile forces when the specimens reached certain elongations.

\section{Test results and analysis}

Test results were shown in Table 2. The best fit lines of the tensile results were got by using the SPSS software, and the tensile results of sample A,B,C could be described by the power law equation, in addition, the tensile results of sample $\mathrm{D}$ and $\mathrm{E}$ could be described by the line equation. The equations of fit lines were shown in Table 3.

\section{Calculate the sizes of compression sleeves}

Calculate the sizes of compression sleeves based on FOM: The volunteer in this study was a 24 years old female, whose height was 
$160 \mathrm{~cm}$ and weight was $49 \mathrm{~kg}$. Began with the wrist radius protuberance of the subject, measurement of circumference of the arm was took every $5 \mathrm{~cm}$ along the arm length direction. The position of measured circumferences was shown in Figure 1. For the formula (8), P (the required garment pressure, that was $24 \mathrm{mmHg}$ or about $18 \mathrm{hPa}$, which was considered as the best pressure value to control and prevent the hyperplasia scars by the other researchers, ${ }^{16} \mathrm{G}_{1}$ (the circumference of the limbs could be measured) and $\mathrm{W}(50 \mathrm{~mm})$ were known, F that was the fabric tension with the fabric width was $\mathrm{W}$ could be calculated. Furthermore, $\mathrm{F}$ was substituted into the regression equations in Table 3 , and fabrics' tensile elongations $\varepsilon$ could be calculated; then used formulas (10) and (11), the sizes of arm sleeve $1_{0}$ could be got. The sizes of the five sleeves (specimens) were shown in Figure 2.

$$
\varepsilon=\frac{l_{1}-l_{0}}{l_{0}}
$$

Table I Parameters of the samples
Where, $\varepsilon$ was the elongation (\%), $1_{0}$ was the original length of the specimen $(\mathrm{cm}), 1_{1}$ was the length of the specimen after being stretched $(\mathrm{cm})$.

$$
f=\frac{l-l_{0}}{C}=\frac{\Delta l}{l_{0}+\Delta l}=\frac{\varepsilon}{1+\varepsilon}
$$

Where, $\mathrm{f}$ was the reduction factor, $\mathrm{c}$ was the arm circumference $(\mathrm{cm}), 1_{0}$ was the size of arm sleeve $(\mathrm{cm}), 1$ was the size of arm sleeve worn on the arm $(\mathrm{cm}), \Delta \mathrm{l}$ was the difference between 1 and $\mathrm{l}_{0}(\mathrm{~cm}), \varepsilon$ was the elongation of the fabric (\%).

The reduction factors of the five specimens could be calculated based on formulas (11). The reduction factors were as follows in Table 4. As shown in Table 4, except for the reduction factors of sample $\mathrm{C}$ and D were a little beyond the range of (0.1-0.2), which were recognized as the reasonable value for making compression garments by the other

\begin{tabular}{|c|c|c|c|c|c|}
\hline Sample & $\mathbf{A}$ & B & C & D & $\mathbf{E}$ \\
\hline Structure & Tricot stitch & Tricot stitch & Tricot stitch & Mesh & Tricot stitch \\
\hline Gram Weight(g.m-2) & 225.48 & 263.03 & 287.9 & 212.82 & 183.75 \\
\hline Pc (wales. $\left.5 \mathrm{~cm}^{-1}\right)$ & 130 & 150 & 125 & 70 & 90 \\
\hline Pw (courses. $\left.5 \mathrm{~cm}^{-1}\right)$ & 100 & 130 & 135 & 70 & 140 \\
\hline Composition (nylon/ spandex) & $70 / 30$ & $70 / 30$ & $65 / 35$ & $70 / 30$ & $60 / 40$ \\
\hline
\end{tabular}
researchers, ${ }^{17}$ other reduction factors were all in the reasonable range.

Table 2 Test results

\begin{tabular}{lllllll}
\hline Samples & \multicolumn{7}{l}{ Unit load at a certain elongation $\left(\mathbf{c N ~} \mathbf{c m}^{-1}\right)$} \\
\cline { 2 - 7 } & $\mathbf{1 0 \%}$ & $\mathbf{2 0} \%$ & $\mathbf{3 0} \%$ & $\mathbf{4 0 \%}$ & $\mathbf{5 0 \%}$ & $\mathbf{6 0 \%}$ \\
\hline A & 42.98 & 79.66 & 105.41 & 130.83 & 155.65 & 174.31 \\
B & 31.8 & 65.72 & 91.6 & 115.87 & 124.37 & 152.9 \\
C & 13.44 & 32.87 & 59.39 & 96.44 & 120.41 & 139.06 \\
D & 40.43 & 71.03 & 91.11 & 124.45 & 153.46 & 178.73 \\
E & 20.16 & 57.06 & 78.69 & 101.94 & 133.48 & 159.72 \\
\hline
\end{tabular}

Then, each paper pattern was done based on the calculated sizes, and flat seaming machine and over lock sewing machine were using for sewing cuff and sleeve body respectively. Compression sleeve was shown in Figure 3.

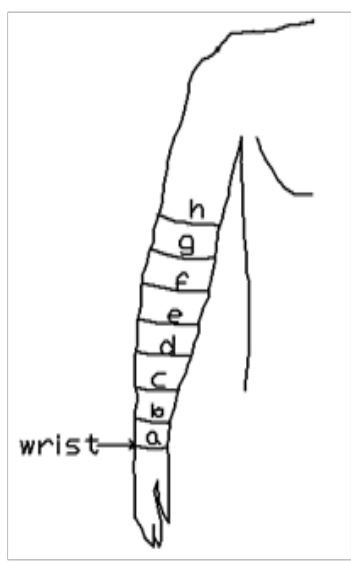

Figure I The positions of measured circumferences.
Table 3 Regression equations

\begin{tabular}{lll} 
Samples & Regression equations & Relative coefficient \\
\hline A & $F=253.963 \times\left(\varepsilon^{\wedge} 0.748\right)$ & 0.999 \\
B & $F=228.184 \times\left(\varepsilon^{\wedge} 0.815\right)$ & 0.996 \\
C & $F=300.37 \mid \times\left(\varepsilon^{\wedge} \mid .349\right)$ & 0.993 \\
D & $F=277.755 \varepsilon+12.654$ & 0.997 \\
E & $F=271.519 \varepsilon-3.192$ & 0.996
\end{tabular}


Calculate the sizes of compression sleeves based on FOL: According to the formula (9) of FOL, samples' elastic moduli should be obtained first. The elastic moduli of the five fabrics were obtained from the tensile results, and were shown in Table 5. Required pressure $(24 \mathrm{mmHg})$ and the other parameters were incorporated in the formula (9), and then $\varepsilon$ of different positions of the arm was obtained. At last, the sizes of the compression sleeves were calculated, and were shown Table 4 Reduction factors of samples base on FOM in Figure 4. The reduction factors of the five samples were calculated based on the calculated sizes, reduction factors were shown in Table 6. As shown in Table 6, all the reduction factors had exceeded the maximum reduction factor 0.2 ; therefore the compression sleeves designed by those sizes were too small to put on. So, the formula of Leung Laplace law (FOL) was not suitable for designing compression sleeves.

\begin{tabular}{lccccc}
\hline Samples & Sample A & Sample B & Sample C & Sample D & Sample E \\
\hline Reduction factor & $0.09-0.14$ & $0.12-0.18$ & $0.19-0.24$ & $0.17-0.23$ & $0.15-0.20$ \\
\hline
\end{tabular}

Table $\mathbf{5}$ The elastic moduli of five samples ( $\mathrm{MPa})$

\begin{tabular}{llllll}
\hline Samples & Sample A & Sample B & Sample C & Sample D & Sample E \\
\hline Elastic modulus & 0.738 & 0.618 & 0.367 & 0.565 & 0.634 \\
\hline
\end{tabular}

Table 6 Reduction factors of samples base on FOL

\begin{tabular}{lccccc}
\hline Samples & Sample A & Sample B & Sample C & Sample D & Sample E \\
\hline Reduction factor & $0.38-0.59$ & $0.43-0.64$ & $0.50-0.70$ & $0.45-0.66$ & $0.42-0.63$ \\
\hline
\end{tabular}

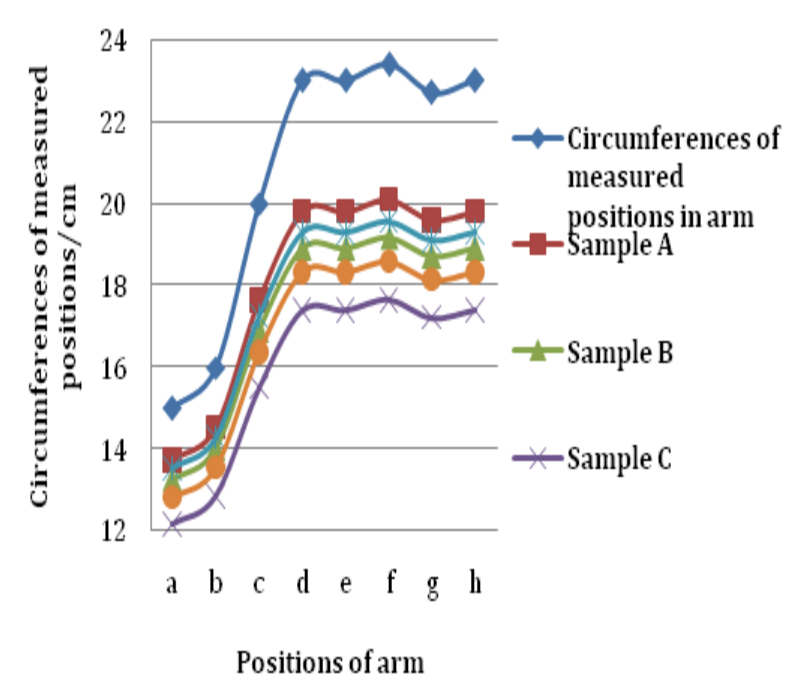

Figure 2 The sizes of the five specimens based on FOM $(\mathrm{cm})$.

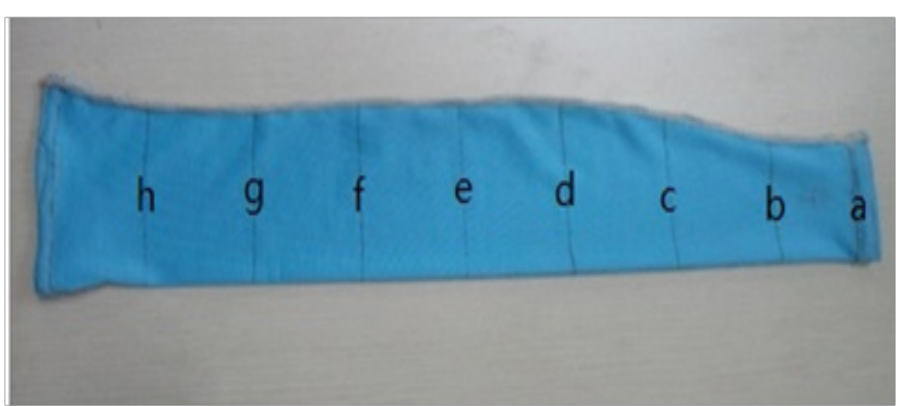

Figure 3 Compression sleeve.

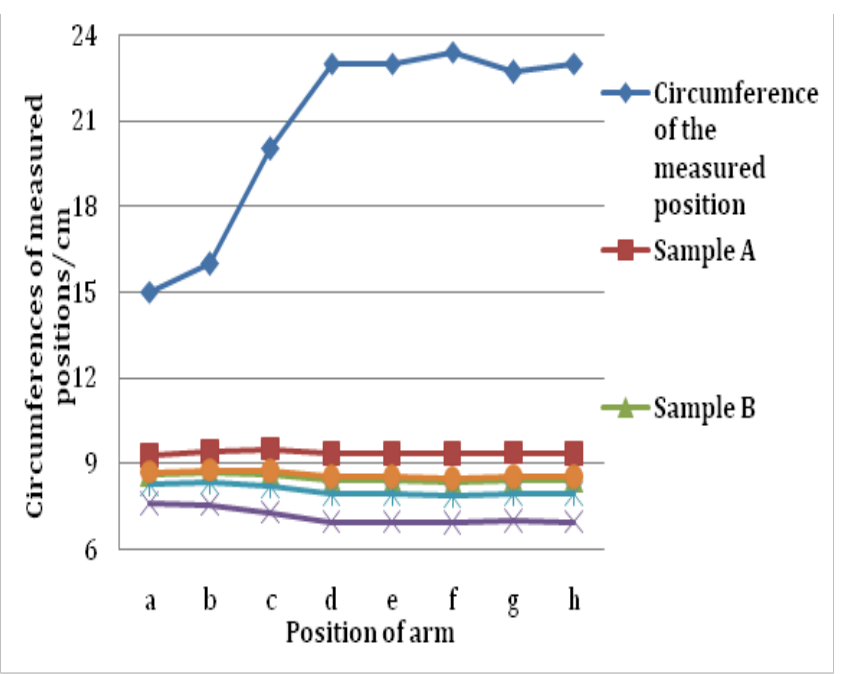

Figure 4 The sizes of the five specimens based on FOL.

\section{Test of garment pressure}

\section{Experimental equipment}

In this study, Flexi force A201 pressure sensor was used for testing the compression sleeve's pressure. The sensor consists of two layers of polyester film and its thickness is only $0.208 \mathrm{~mm}$. Each film contains silver conductor and pressure sensitive conductive materials. The flexibility of the sensor is good, and it is easy to bend. So it can detect the pressure exerted by the compression sleeves conveniently and accurately. 


\section{Experimental process}

Firstly, calibrated the sensors, the calibrate processes as follows: (1) applied continuous load with known weight on the sensor, the loads were $10 \mathrm{~g}, 20 \mathrm{~g}, 30 \mathrm{~g}, 50 \mathrm{~g}$ and $70 \mathrm{~g}$, and every loading time would keep for 1 minute; (2) recorded the output voltage value during the loading; (3) calculated the best regression equation between the input force value and the output voltage.

Before measuring the pressure, compression sleeve was put on the subject with the legs of the subject straight and hands hanging down in a natural position for five minutes then tested the pressure exerted by the compression sleeve on the different points of the arm. Four points (up, down, left and right) at regular intervals were measured in circumferential lines, and three values were recorded at every point.

\section{Test results and analysis}

Took voltage average value of four locations (that contain $(4 \times 3)$ voltage values) as the final voltage value of one certain circumference. The final voltage values of every certain circumference were incorporated in the regression equation of the force and the voltage, and then combined with the sensor area (about $0.19624 \mathrm{~cm}^{2}$ ), the garment pressures exerted by the compression sleeves were calculated and shown in Figure 5.

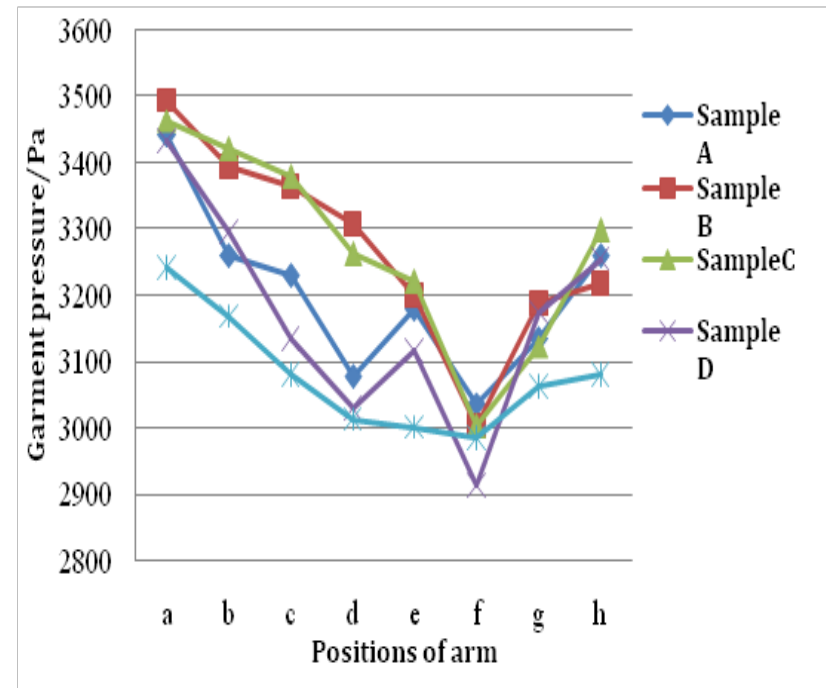

Figure $\mathbf{5}$ The garment pressure exerted by the five pressure sleeves on different position of arm.

According to previous studies, ${ }^{16}$ the ideal range of garment pressure for curing the hyperplasia scar was (20-25) $\mathrm{mmHg}$ which was equal to (2666.40-3333.00) Pa. As shown in the Figure 5, the pressures exerted by the five samples were all among the scope of the ideal pressure although there was some difference from the designed pressure value (3199.68Pa). The test results also showed that the pressures at the wrist was bigger than those of other positions on the arm; the reason might be that the wrist radius protuberance was a prominent position, and the sizes calculated was based on the whole circumference of every position, which led to the bigger pressure at certain part. The pressures at the elbow were less than those of other positions on the arm, which might result from the sunken place at the elbow where the pressure sensor was not well contact with the compression sleeve very well. From the overall trend of the pressure distribution, it could be found that the pressures exerted by the sample $\mathrm{C}$ and $\mathrm{B}$ were a little larger than those of the sample A and D, and the pressure exerted by the sample E was the least. This might be due to the level of the garment pressure was also affected by the fabric gram weight. In the case of a certain required garment pressure, the less gram weight of the sample was, the smaller the pressure would impose based on the deformed Laplace formula researched by former research.

\section{Conclusion}

The garment pressure exerted by the five sleeves which were designed and manufactured based on the FOM were all in the scope of the ideal pressure although there were some difference from the designed pressure value. Therefore, this method could be used for designing and manufacturing compression sleeves accurately. The sizes of compression sleeves designed based on the FOL were too small to put on, so the formula of Leung developed from Laplace law was not suitable for designing the compression sleeves.

The distribution tend of the garment pressure exerted by the five compression sleeves manufactured by five different samples were almost consistent. The pressure at the wrist was bigger than the other positions on the arm because there was a prominent position at the wrist radius protuberance, at the same, the wrist just at the cuff which had two layers fabric. The pressure at the elbow was less than the pressure at the other positions on the arm, which might lead by the sunken place at the elbow where the pressure sensor might not contact with the compression garment very well. Therefore a pressure pad might be put at the sunken place at the elbow to increase the pressure, so that it could achieved the required pressure value.

There were some shortcomings in this study; however, for example, the kinds of the compression garment fabric were not enough, which leads to the impact of the fabric structures on the garment pressure were not deeply analyzed. All the shortcomings will be researched in our future studies.

\section{Acknowledgements}

This work is supported by NSFC (Natural Science Foundation of China) under Grant No. 51403154.

\section{Conflict of interest}

Author declares there is no conflict of interest in publishing the article.

\section{References}

1. Wang L, Felder M, Cai JY. Study of Properties of Medical Compression Garment Fabrics. J Fiber Bioengineering \& Informatics. 2011;4(1):15-22.

2. Widgerow AD. Wound Healing Southern Africa-Hypertrophic burn scar evolution and management: review. Wound Healing Southern Africa. 2013;6(2):79-86.

3. Zurada JM, Kriegel D, Davis IC. Topical treatments for hypertrophic scars. J Am Acad Dermatol. 2006;55(6):1024-1031.

4. Kim JY, Willard JJ, Supp DM, et al. Burn Scar Biomechanics after Pressure Garment Therapy. Plastic \& Reconstructive Surgery. 2015;136(3):572-581.

5. Li Tsang CW, Feng B, Huang L, et al. A histological study on the effect of pressure therapy on the activities of myofibroblasts and keratinocytes in hypertrophic scar tissues after burn. Burns. 2015;41(5):1008-1016. 
6. Anand SC, Govarthanam KK, Gazioglu D. A study of the modelling and characterization of compression garments for hypertrophic scarring after burns. Part 1: modelling of compression garments. J Textile Institute. 2013;104(7):661-667.

7. Wang YR, Zhang PH. Garment Pressure Measurement and its Sensor Technology. China Textile Leader. 2008;(7):154-155.

8. Silfen R, Amir A, Hauben DJ, et al. Effect of facial pressure garments for burn injury in adult patients after orthodontic treatment. Burns. 2001;27(4):409-412.

9. Rappoport K, Müller R, Flores Mir C. Dental and skeletal changes during pressure garment use in facial burns: A systematic review. Burns. 2008;34(1):18-23.

10. Cheng JCY, Evans JH, Leung KS, et al. Pressure therapy in the treatment of post-burn hypertrophic scar-a critical look into its usefulness and fallacies by pressure monitoring. Burns. 1984;10(3):154-163.

11. Ng SF, Hui CL. Pressure Model of Elastic Fabric for Producing Pressure Garments. Textile Research J. 2001;71(3):275-279.
12. Thomas $\mathrm{S}$. The use of the Laplace equation in the calculation of sub-bandage pressure. EWMA journal. 2003;3(1):21-23.

13. Macintyre L, Baird M, Weedall P. The study of pressure delivery for hypertrophic scar treatment. International J Clothing Science \& Technology. 2004;16(1/2):173-183.

14. Maklewska E, Nawrocki A, Ledwoń J, et al. Modelling and Designing of Knitted Products Used in Compressive Therapy. Fibres \& Textiles in Eastern Europe. 2006;14(5):111-113.

15. Leung WY, Yuen DW, Ng SP, et al. Pressure prediction model for compression garment design. J Burn Care Res. 2010;31(5):716-727.

16. Anand SC. Bandaging and Pressure Garments: An Overview. Medical \& Healthcare Textiles. 2010. p. 257-262.

17. Macintyre L, Baird M. Pressure garments for use in the treatment of hypertrophic scars-an evaluation of current construction techniques in NHS hospitals. Burns. 2005;31(1):11-14. 